\title{
Comprehensive training programme for judo players nine plus 9+: possible lower limb primary injury prevention
}

\author{
Nikos G. Malliaropoulos ${ }^{1}$ \\ Michael Callan² \\ Janine Johnson ${ }^{3}$ \\ 1 National Track \& Field Centre, Sports Injury Clinic, \\ Sport Medicine Clinic of SEGAS, Thessaloniki, \\ Greece \\ 2 Judo Research Group, Department of Life Sci- \\ ences, Anglia Ruskin University, Cambridge, UK \\ 3 Judospace Educational Institute, Bath, UK
}

Corresponding author:

Nikos G. Malliaropoulos

National Track \& Field Centre, Sports Injury Clinic, Sport

Medicine Clinic of SEGAS, Thessaloniki

Asklipiou 17, 54639, Thessaloniki, Greece

E-mail: contac@sportsmed.gr

Dear Editor judo is a popular and commonly practiced Japanese martial art and an Olympic combat sport. Judo has its roots in Japanese unarmed fighting styles from the sixth century, the forerunners to ju-jitsu, and was founded by Jigoro Kano in 1882 in Tokyo $^{1}$. It is a martial art, a physical education, an Olympic sport, and a way of life $e^{2,3}$. It is characterized by a great number of techniques and its history, culture and philosophical basis. Currently the International Judo Federation estimates 20 million participants ${ }^{4}$.

Since the first time judo was included in the Olympic Games, the skill level of judo players has been dramatically increasing along with the rate and severity of injuries ${ }^{5}$. Judo is a contact sport; there is a relative risk for an injury as in every other sport. There is little literature on the frequency and seriousness on Judo injuries, it is important to continue to analyse the injuries that do occur.

The most injured body areas were ${ }^{6}$ : knee (26.3\%), shoulder $(21.8 \%)$, fingers (17.3\%), and ankle (10.0\%).

\section{Severity}

A study indicates that $49 \%$ of injuries happen to the judoka in competition situations, $43.6 \%$ in training situations and $3.6 \%$ during the physical conditioning 5,6 Conflicting research shows that only $30 \%$ of injuries have genesis on competition events, comparing the $70 \%$ that happen during training situations, the latter results are supported by evidence that shows that judoka spend more time training than competing ${ }^{7}$. In future it may be beneficial to measure the prevalence and severity of injuries between a set amount of hours training and competition; for example a comparison between 500 hours of competing and 500 hours of training to determine a more accurate account of injuries in judo.

Existing studies state that the most common mechanism of injury happens during the standing situation (tachi-waza), when the athlete is being thrown or fighting for the grips, less when the athlete tries to throw the opponent or during the ground fight (newaza $)^{8}$. This probably relates to the fact that during the judo match the athlete spends more time in tachiwaza (56\% of the time of the match) than in ne-waza $(18 \% \text { of the time of the match })^{9}$. Injuries occur more frequently during standing practice (tachi-waza randori) especially with opposite gripping (kumikata). Standing techniques (tachi-waza), such as; ipponseoi-nage for the thrown athlete (uke) with a $23 \%$ incidence, tai-otoshi for the throwing athlete (tori), with $22 \%$ incidence, and uchi-mata, with $9 \%$ incidence $^{6}$. In addition, Koshida et al. found that being attacked with o-soto-gari was revealed as the leading cause for knee injuries especially the anterio-cruciate-ligament $(A C L)$ injury incidence among the subjects $(16.8 \%)^{10}$.

Injuries in sports constitute a considerable problem for the athlete, the family, the club, and, given the popularity of sports participation, for society at large. Health consequences of sports injuries are seen not just in the short term but also in the dramatic increase in the risk of early osteoarthritis ${ }^{11,12}$.

Programmes have been implemented in other sports to prevent similar injuries. Ettlinger et al. developed an education program for the prevention of $A C L$ injuries in alpine skiing to make skiers recognize the dangerous situation for the injury. It was reported that the rate of the ACL injury in highly skilled alpine skiers decreased through the use of the education program ${ }^{13}$. Similarly in basketball and soccer lower limb injuries have been reduced through a neuromuscular training program developed for ACL injury prevention ${ }^{11,14-17 .}$

To the authors knowledge there is no such prevention programme formally implemented within judo and without one it is unclear whether the incidence of lower limb injuries in judo could be reduced. 
The high occurrence of lower limb injuries and lack of prevention programmes thus led us to develop and design a structured training programme "The Judo $9+"$. It includes a set of structured sports specific exercises that make it suitable as a comprehensive warm-up programme for training and competition. The benefits of implementing such a program should be fewer injuries, lower cost due to a reduction in injuries that will need medical management either conservative or surgical, and better performance.

The programme is intended for use by the full spectrum of active judoka post puberty. These judoka will typically range from under $55 \mathrm{~kg}$ to over $100 \mathrm{~kg}$ for men, and under $44 \mathrm{~kg}$ to over $78 \mathrm{~kg}$ for women.

\section{Validity reliability and implementation}

With knowledge of the high prevalence of lower limb injuries in judo and the severity of the impact they can have on the athletic and day to day life of a judoka it is important to consider current methods in place that aim to minimise such occurrences; in order to assess their effectiveness and implementation. To the authors knowledge there is no formal implementation of a lower limb injury prevention protocol in judo, this may be surprising considering the accumulating wealth of evidence that shows targeted training can reduce injury risk ${ }^{14,18,19}$.

From the literature on other sports, possibly one of the most well known targeted training programmes is "The 11+" programme developed by a specialised research team for the International Football Federation (FIFA) in 2006. The 11+ was developed after identifying and addressing injury risks in footballers and has since been found effective in male and female football players ${ }^{12,20}$.

Programmes similar to the $11+$ have been developed to satisfy sport specific mechanical demands and shown to be effective in other sports, and can reduce injury risk ${ }^{11,13,16,17,21}$. This led the authors to conclude there is a need to develop a programme specific to the needs of judoka.

\section{Materials and methods}

In developing this protocol the authors acted according to international ethical standards ${ }^{22}$.

\section{Judo 9+}

The intervention consists of sports specific exercises focusing on, balance, dynamic stabilisation, and core stability, utilising static and dynamic movements ${ }^{14,23}$. The "Judo 9+" has three parts with a total of 9 exercises which should be performed in the specified sequence at the start of each training session, as part of the warm-up routine.

The exercises are performed on a standard Judo mat (10 metres $\times 10$ metres) travelling up and down the mat.

\section{The Judo 9+ routine}

1. Running forwards with shoulder contact between partners (pushing each other) (Fig. 1).

a. Left and Right

b. Progression: Gradual increase of force and/or speed

2. Running forwards with shoulder contact between partners (pulling each other) (Fig. 2).

a. Left and Right

b. Progression: Gradual increase of force and/or speed

3. Running backwards with shoulder contact between partners (pushing each other) (Fig. 3).
a. Left and Right
b. Progression: Gradual increase of force and/or speed

4. Running backwards with a partner with shoulder contact between partners (pulling each other) (Fig. 4).

a. Left and Right

b. Progression: Gradual increase of force and/or speed

5. Walking lunges. (Fig. 5).

6. Forward jump. (Fig. 6A-D).
a. Double leg.
b. Progression: Single leg hop; left and right.

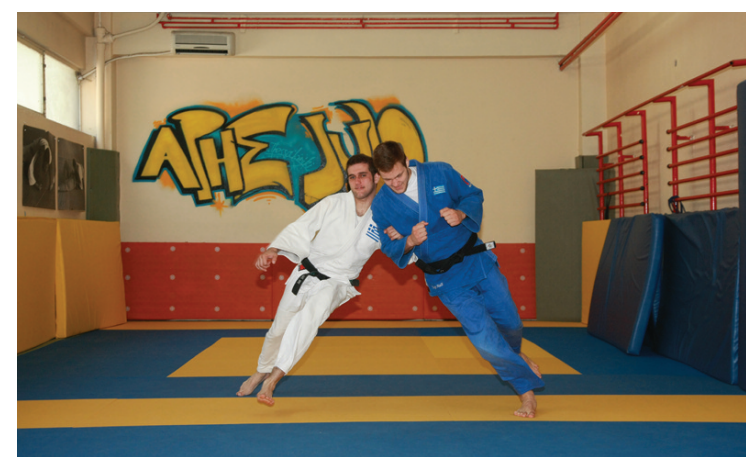

Figure 1. Running Forwards (Pushing each other) with a Partner- shoulder contact running.

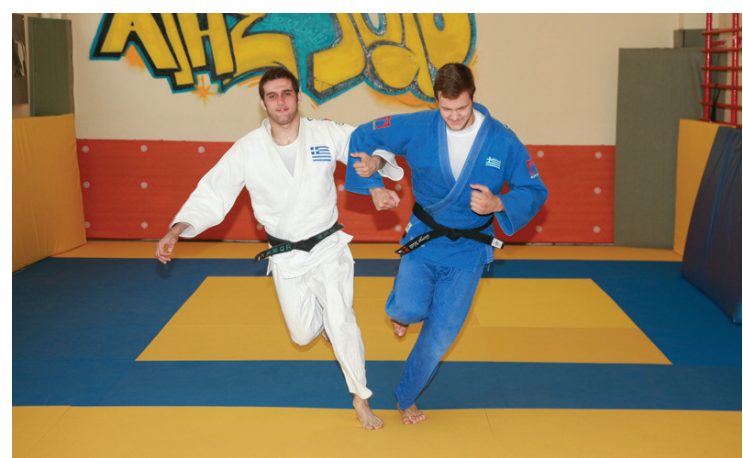

Figure 2. Running Forwards (Pulling each other) with a Partner- shoulder contact running (Pulling each other). 


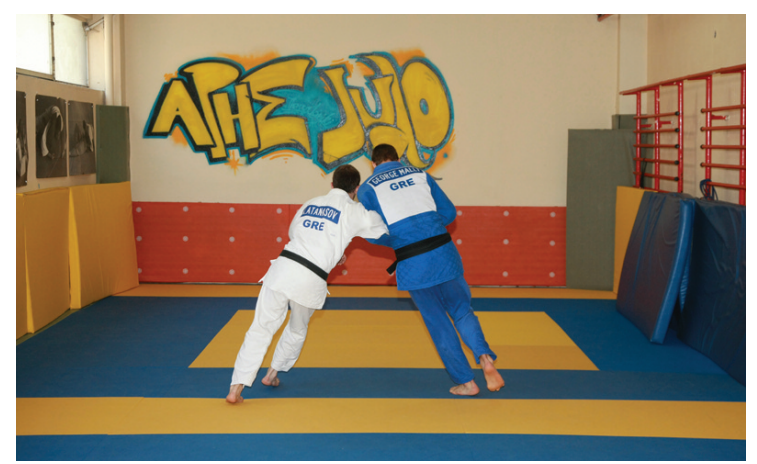

Figure 3. Running Backwards (Pushing each other).

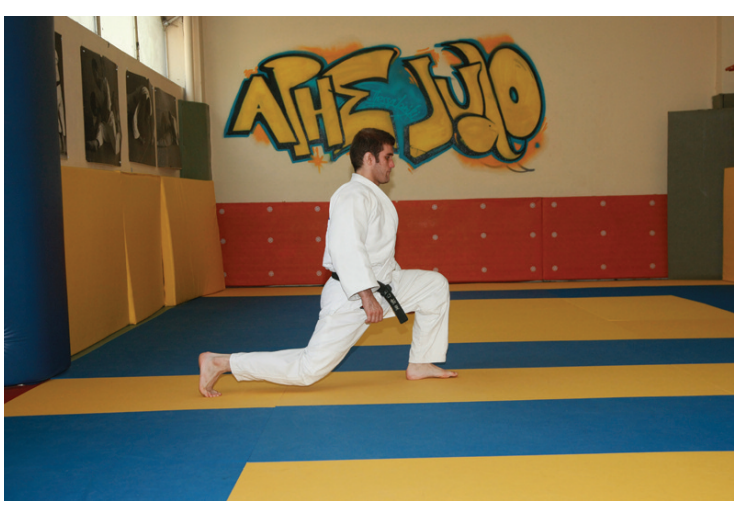

Figure 5. Walking lunges 1.

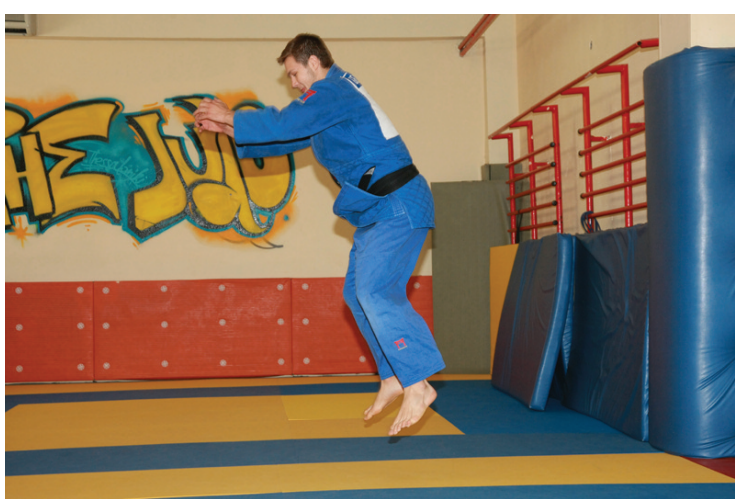

Figure 6b. Two legs hops 2.

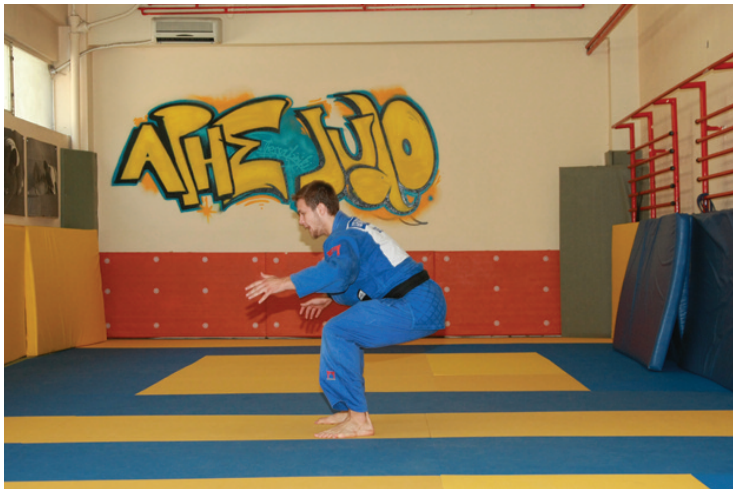

Figure 6d. Two legs hops 4.

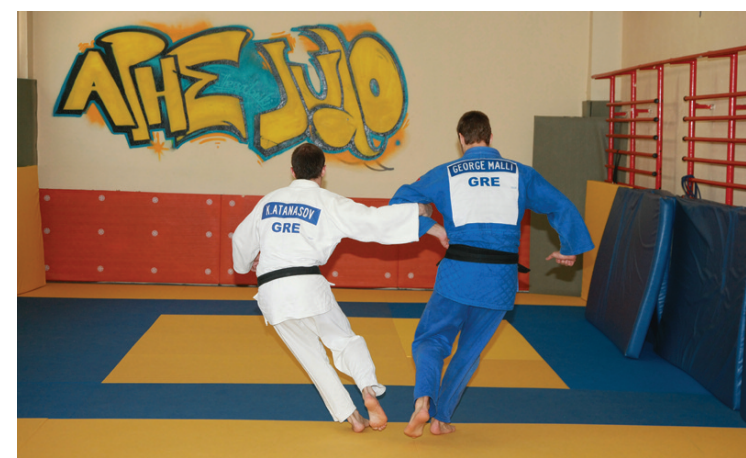

Figure 4. Running Backwards (Pulling each other).



Figure 6a. Two legs hops 1.

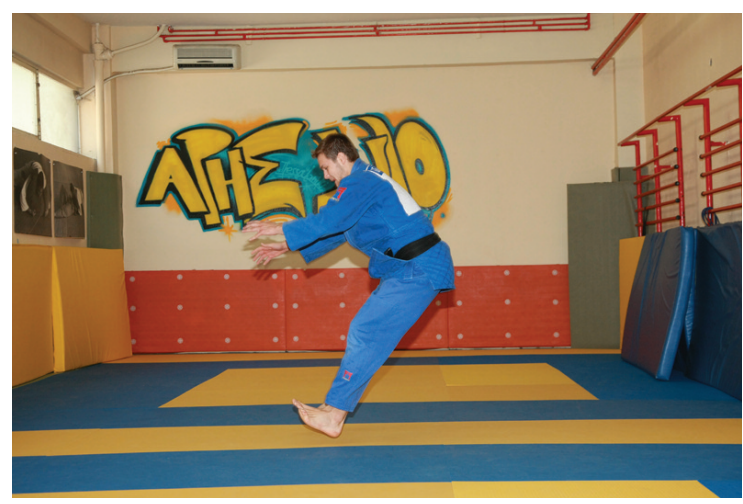

Figure 6c. Two legs hops 3.

7. Hops 'across' line. (Fig. 7A-C).

a. Double leg

b. Progression: Single leg; left and right.

8. O-soto-gari moving forwards; left and right. (Fig. $8 \mathrm{~A}, \mathrm{~B})$.

9. O-uchi-gari moving forwards; left and right. (Fig. 9A,B).

\section{Implementation of Judo 9+}

It is suggested that the routine is carried out by players at least twice a week as part of their training session. Where there are multiple options ('a' and 'b') all players should start on level 'a'. Once competent at 


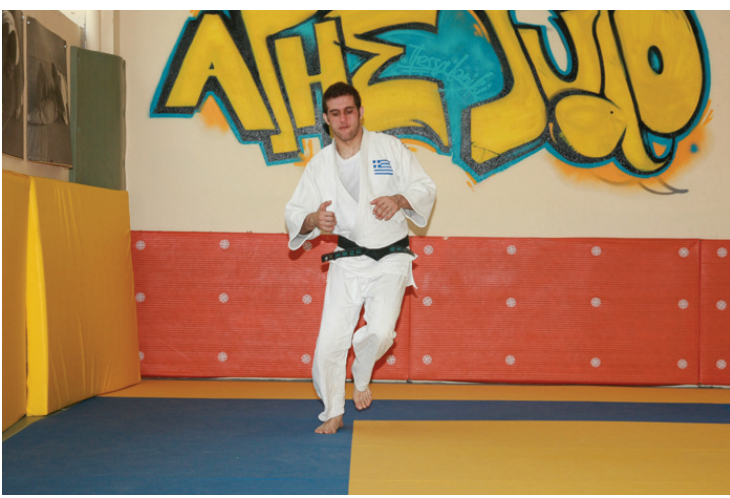

Figure 7a. One legs hops Cross 1.

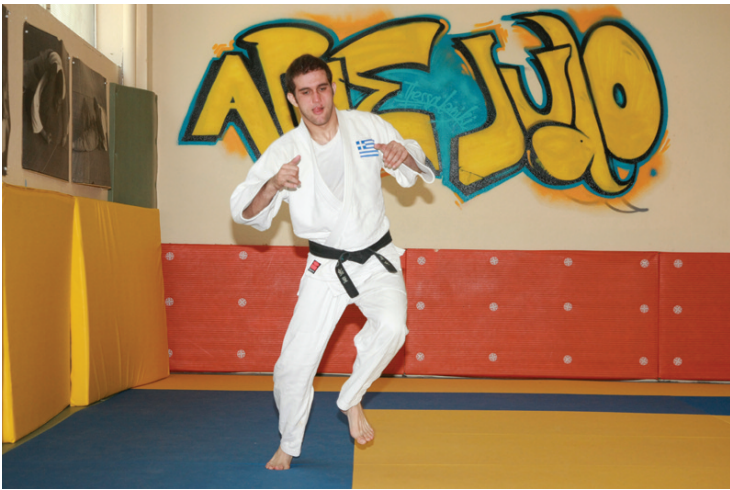

Figure 7c. One legs hops Cross 3.

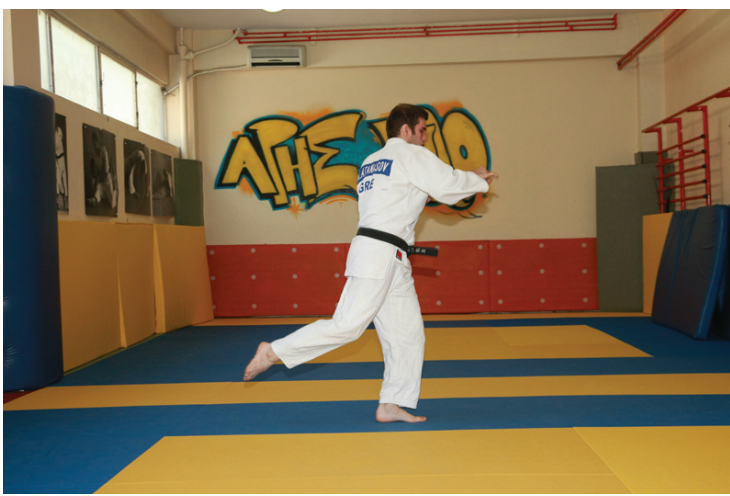

Figure 8b. O-soto-gari 2.

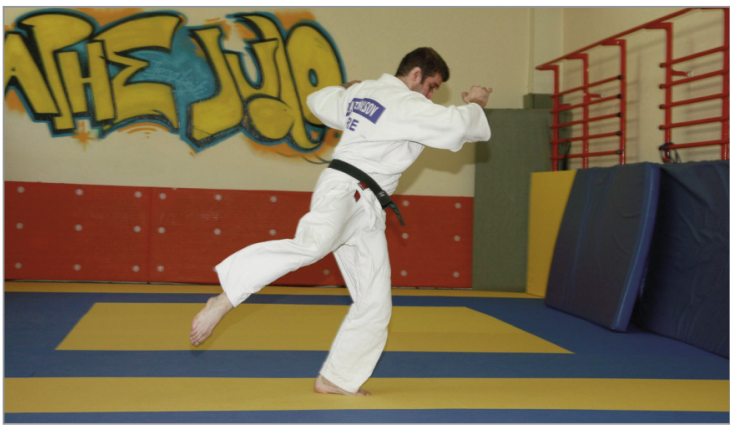

Figure 9b. O-uchi-gari.

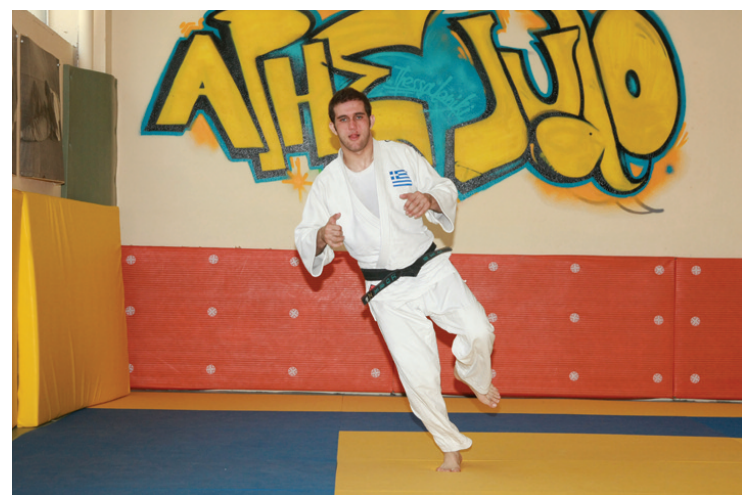

Figure 7b. One legs hops Cross 2.

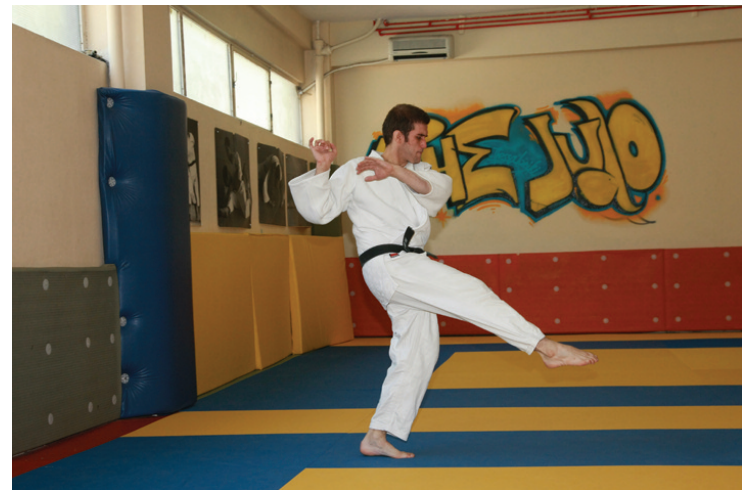

Figure 8a. O-soto-gari 1.

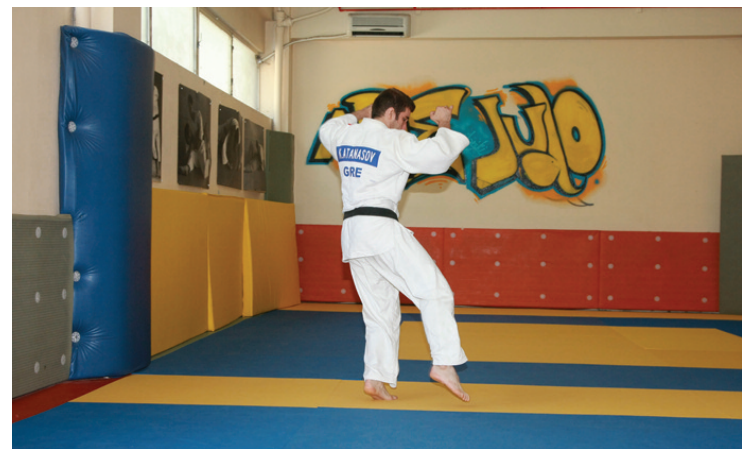

Figure 9a. O-uchi-gari.

this level, players can progress with level 'b'. Only when an exercise can be performed without difficulty is the player competent enough to progress to the next level. Assuming the routine is utilized twice a week, this progression should occur between three to four weeks of practice.

Crucial to the programme is the execution of correct technique during all of the exercises. Full attention should be paid to correct posture and good body control, including knee-over-toe position and soft landings. Additional key features are hip control and knee alignment, which avoids excessive knee valgus during both static and dynamic movements ${ }^{18,24}$. 


\section{Important}

Correct technique is of great importance, therefore the coach should supervise the programme and correct the players if necessary.

\section{Discussion}

The Judo $9+$ is a routine that is easy to incorporate into the warm-up programme of judo athletes in both a competitive and training environment. It has been designed to prevent lower limb injuries to judo athletes with the intent of reducing medical costs and injury rates; consequently enhancing performance.

\section{Injuries in Judo}

The risk of knee injury is greater than that of any other anatomical structure $8,25-28$. On the 4-year epidemiological research in Finland, Kujala et al. reported that the knee injury accounted for approximately $20 \%$ of all the injuries in judo ${ }^{7}$.

Miyazaki et al. also showed that 133 out of 145 collegiate freshman judokas had had a previous history of knee injury and 94 of them had also reported multiple episodes of knee injury in the pre-season physical examination ${ }^{28}$.

The most commonly reported mechanism of knee injuries in judo was the direct contact to the lower extremity. This is the type of movement utilized when the opponent attempts to throw with o-soto-gari, kosoto-gari, and harai-goshi.

The athletes who were injured by being attacked in the role of uke (the receiver) with $\mathrm{O}$-soto-gari, reported they had felt the valgus collapse in the knee joint at the point of contact. Most subjects also reported that the opponent forcefully rotated their upper body against their static, weight bearing leg, thus causing the knee to twist ${ }^{10}$.

With the knowledge from such research the aims of the Judo 9+ were determined. Thus being to teach correct knee control and core stability during commonly performed standing techniques in judo tachiwaza, and to train the body's muscles and reactions in how to deal with situations that may occur when throws such as o-soto-gari, harai-goshi and tai-otoshi are performed correctly and incorrectly.

O-soto-gari and harai-goshi are similar techniques which involve sweeping the opponents leg with direct leg on leg contact. In the preparatory phase of the throw, the centre of mass of the judoka being attacked (uke) needs to be moved in medial-lateral direction, resulting in the shift of body weight on to one leg. At this point the attacker tries to break uke's balance, an action known as kuzushi29. Simultaneously, the judoka who is attacking (tori) needs to place his body in the correct position in order to execute a successful throw, an action known as tsukurio. In performing common techniques in judo such as o-soto-gari and harai-goshi, should the attacking judoka fail to balance themselves correctly, or misplace/misjudge uke's body position during the preparatory phase, their own knee could sustain a large valgus stress combined with a rotational force. This is considered to put the athlete at high risk for ACL injury and is considered to be a key mechanism for causing ACL injury ${ }^{10}$.

It is noteworthy that ACL injuries may also occur when attempting to attack with tai-otoshi. This technique requires tori to break uke's balance in a forward direction, then placing their leg in front of uke's leg, causing uke to trip over tori's leg to the front. In the event of an ACL injury, the valgus stress may have been applied to tori's knee by uke's leg coming down in direct contact on to tori's extended knee. The risk of this injury by attempting tai-otoshi appears to be higher when the judoka's knee joint is in full or almost full extension during the preparatory phase.

Therefore, as tori and as uke, it appears important to practice the exercise of balancing on one leg with frequency during training. The warm-up is an ideal time to perform this kind of sports specific movement, as a feed back to the lower limb for strength and proprioception.

\section{Related lower limb warm-up routines}

Ettlinger et al. developed an education program for ACL injury prevention in Alpine Skiing to help skiers recognise the dangers of injury and reported that the rate of ACL injury occurrence in high skilled alpine skiers decreased through the use of the education program $^{13}$.

This rationale is justified by data from studies on the mechanisms of anterior cruciate ligament injuries ${ }^{31-33}$. These studies indicate that athletes could benefit from avoiding the knee to sag medially in sport specific movements during sudden changes of speed, or during tackles by opponents.

The focus on balance, neuromuscular control, core stability, hip control and knee alignment which collectively avoid excessive knee valgus during sports specific dynamic movements, is a feature of earlier intervention studies ${ }^{11,18,34}$.

Prevention of injuries is a major challenge in sport, including judo ${ }^{35}$. The view of the authors is that this responsibility rests mainly with the sports medicine physicians working closely with the coaches. The coach education programme of the European Judo Union has recently (2013) introduced additional sports medicine information into their curriculum.

\section{Further recommended steps to implement Judo 9+}

Step 1: Pilot test of the program to test the intervention, i.e. what works, what does not work, what do coaches say, are the levels feasible etc.

Step 2: The translation of this pilot into a slightly adapted program, building on the pilot, step 1 . 
Step 3: Wider implementation and Randomized Control Trials (RCT) implementing Judo 9+ program.

\section{Conclusion}

We think that is time to move from the so called "traditional" warm-up used in judo, such as running around the mat and performing static exercises. It is time to move from static to functional, from general to sports specific, from single performing to a couple performing warm-up.

The Judo 9+ warm-up prevention program is Sports Specific, multifaceted and addresses many factors such as (strength, balance, awareness of vulnerable hip and knee positions, technique, and landing) that could be related to the risk of Lower Limb injuries.

As a next step the authors plan to conduct Randomized Control Trials (RCT) implementing the Judo 9+ program.

\section{Summary box}

\section{"What this paper adds"}

This is the first paper to propose a structured sports specific program to be included within the warm-up routine, designed to prevent lower limb injuries in Judo. Emphasises the necessity of moving from the currently "traditional" warm-up routine followed in judo training, towards a functional and couple performing programme.

\section{"How might it impact on clinical practice in the near future?"}

By presenting the judo 9+ training program we have a tool in hand to prevent Lower Limb injuries in judo, leading the future research (pilot, RCT).

Federations through their Medical, Education and Coach Committees, government through their coaching education programs, Sports Medicine Physicians and sporting authorities need to further adopt and encourage such strategies in order to further prevent sports injuries, fewer costs, and better performance. Further prevention programs incorporated in Judo training must further developed and implemented in terms of preventing Upper Limp injuries as well.

\section{Acknowledgments}

To all the Aris Judo Club athletes for their contribution on performing the entire warm-up exercises.

\section{Contributorship}

Nikos Malliaropoulos planned the study, and contributed to write the manuscript.
Mike Callan and Janine Johnson contributed to write the manuscript.

\section{References}

1. Watson B. The Father of Judo: A biography of Jigoro Kano. Tokyo: Kodansha International Ltd. 2000:21-24.

2. Matsumoto D. An Introduction to Kodokan Judo: History and Philosophy. London: Hon-no Tomosha. 2001:10-11.

3. Callan MJ. Elite Sport and Education Support Systems: A Case Study of the Team Bath Judo Programme at the University of Bath. PhD thesis. University of Bath. 2008.

4. http://www.worldjudoday.com/en/WhatisJudo-57.html (Accessed May,2013).

5. Pierantozzi E, Muroni R. Judo high level competitions injuries. MEDit J MUSC SURV 2009;17:26-29.

6. Souza M, Monteiro H, Del Vecchio F, et al. Referring to judo's sports injuries in Sao Pãulo state championship. Science \& Sports. 2006;21(5):280-284.

7. Green CM, Petrou J, Fogarty-Hover C, et al. Injuries among judokas during competition. Scand J Med Sci Sports. 2007;17: 205-210.

8. International Judo Federation. http://judoinfo.com/new/articlesindex/research-and-technical-papers/408-an-evaluation-ofmodern-tendencies-in-solving-judo-fight (Accessed April, 2013)

9. Koshida S, Deguchi T, Miyashita K, et al. The common mechanism of anterior cruciate ligament injuries in judo: a retrospective analysis. Br J Sports Med. 2010;44:856-861 .

10. Myklebust G, Engebreten L, Braekken $\mathrm{H}$, et al. Prevention of $A C L$ injuries in female team handball players: a prospective intervention study over three seasons. Clin J Sport Med. 2003;13:71-78.

11. Von Porat A, Roos EM, Roos H. High prevalence of osteoarthritis 14 years after an anterior cruciate ligament tear in male soccer players: a study of radiographic and patient relevant outcomes. Ann Rheum Dis. 2004;63:269-273.

12. Caraffa A, Cerull G, Projetti M, et al. Prevention of anterior cruciate ligament injuries in soccer. A prospective controlled study of proprioceptive training. Knee Surg Sports Traumatol Arthrosc. 1996;4:19-21.

13. Soligard T, Myklebust G, Steffen K, et al. Comprehensive warm-up programme to prevent injuries in young female footballers: cluster randomized controlled trial. BMJ. 2008; 337:a2469.

14. Mandelbaum BR, Silvers HJ, Watanabe DS, et al. Effectiveness of a neuromuscular and proprioceptive training program in preventing anterior cruciate ligament injuries in female athletes: 2-year follow-up. Am J Sports Med. 2005;33: 1003-1010.

15. Olsen OE, Myklebust G, Engebretsen L, et al. Exercises to prevent lower limb injuries in youth sports: cluster randomized controlled trial. BMJ. 2005;330:449.

16. Kujala UM, Taimela S, Antti-Poika I, et al. Acute injuries in soccer, ice hockey, volleyball, basketball, judo, and karate: analysis of national registry data. Brit Med J. 1995;311:1465-1468.

17. Filiare E, Maso F, Degoutte F, et al. Food restriction, performance, psychological state and lipid values in judo athletes. Int J Sports Med. 2001;22:454-459.

18. Onda T, Aruga S, Terao T, et al. The study on injures in university judo players.

19. Nishimura N, Nakamura $Y$, Aruga $S$, et al. Investigation of injuries in athletes. Tokai J Sports Med Sci. 2003;15:60-66. (Japanese)

20. Miyazaki S, Nakamura Y, Yamaji O, et al. Injuries in university judo players. Tokai J Sports Med Sci. 1997;9:9-12. (Japanese)

21. Miyazaki S, Nakamura Y, Sato N, et al. Knee injuries of uni- 
versity judoka in eleven years. Tokai J Sports Med Sci. 2002;14:58-62. (Japanese)

22. Imamura R, Hreljac A, Escamilla RF, et al. A three-dimensional analysis of the centre of mass for three different judo throwing techniques. J Sports Sci Med. 2006;CSSI,122-131.

23. Kano J. Kodokan Judo. Tokyo: Kodansha International Ltd. 1986:37-55

24. Steffen K, Myklebust G, Olsen OE, et al. Preventing injuries in female youth football - a cluster-randomized controlled trial. Scand J Med Sci Sports. 2008;18(5):605-614.

25. Olsen OE, Myklebust G, Engebretsen L, et al. Injury mechanisms for anterior cruciate ligament injuries in team handball: a systematic video analysis. Am J Sports Med. 2004;32:1002-1012.

26. Hewett TE, Myer GD, Ford KR, et al. Biomechanical measures of neuromuscular control and valgus loading of the knee predict anterior cruciate ligament injury risk in female athletes: a prospective study. Am J Sports Med. 2005;33:492-501.

27. Krosshaug T, Nakamae A, Boden BP, et al. Mechanisms of anterior cruciate ligament injury in basketball: video analysis of 39 cases. Am J Sports Med. 2007;35:359-367.

28. Caraffa A, Cerull G, Projetti M, et al. Prevention of anterior cruciate ligament injuries in soccer. A prospective controlled study of proprioceptive training. Knee Surg Sports Traumatol Arthrosc. 1996;4:19-21.
29. Hewett TE, Lindenfeld TN, Riccobene JV, et al. The effect of neuromuscular training on the incidence of knee injury. Am J Sport Med. 1999;27:699-706.

30. Malliaropoulos N, Mendiguchia J, Pehlivanidis H, et al. Hamstring exercises for track and field athletes: injury and exercise biomechanics, and possible implications for exercise selection and primary prevention. Br J Sports Med. 2012;46(12):846851.

31. Ettlinger CF, Johnson RJ, Shaly JE. A method to help reduce the risk of serious knee sprains incurred in alpine skiing. Am $J$ Sports Med. 1995;23:531-537.

32. Besier TF, Lloyd DG, Cochrane JL, Ackland TR. External loading of the knee joint during running and cutting maneuvers. MSSE 2001;33(7):1168-1175.

33. Lloyd DG. Rationale for training programs to reduce anterior cruciate ligament injuries in Australian football. JOSPT 2001;31(11):645-654; discussion 661.

34. Olsen L, Scanlan A, MacKay M, et al. Strategies for prevention of soccer related injuries: a systematic review. BJSM 2004; 38(1):89-94.

35. Padulo J, Oliva F, Frizziero A, Maffulli N. Muscles, Ligaments and Tendons Journal. Basic principles and recommendations in clinical and field science research. MLTJ. 2013;4:250-252. 